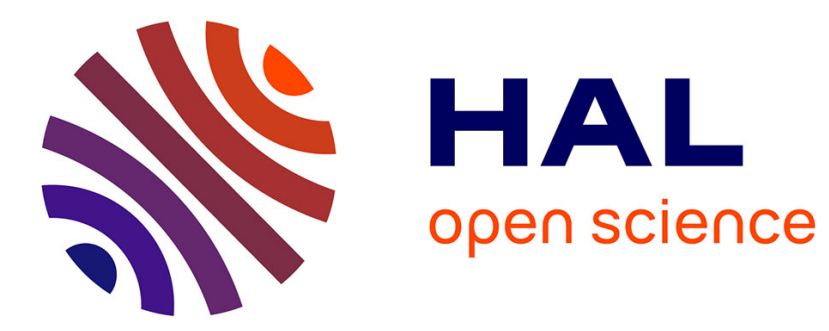

\title{
Piola transformations in second-gradient continua
}

Simon R. Eugster, Francesco Dell'Isola, Roberto Fedele, Pierre Seppecher

\section{To cite this version:}

Simon R. Eugster, Francesco Dell'Isola, Roberto Fedele, Pierre Seppecher. Piola transformations in second-gradient continua. 2022. hal-03454835v2

\section{HAL Id: hal-03454835 \\ https://hal.science/hal-03454835v2}

Preprint submitted on 20 Jan 2022

HAL is a multi-disciplinary open access archive for the deposit and dissemination of scientific research documents, whether they are published or not. The documents may come from teaching and research institutions in France or abroad, or from public or private research centers.
L'archive ouverte pluridisciplinaire HAL, est destinée au dépôt et à la diffusion de documents scientifiques de niveau recherche, publiés ou non, émanant des établissements d'enseignement et de recherche français ou étrangers, des laboratoires publics ou privés. 


\title{
Piola Transformations in Second-Gradient Continua
}

\author{
Simon R. Eugster ${ }^{a, e, *}$, Francesco dell'Isola ${ }^{b, e}$, Roberto Fedele $^{c, e}$, Pierre Seppecher $^{d, e}$ \\ ${ }^{a}$ Institute for Nonlinear Mechanics, University of Stuttgart, Stuttgart, Germany \\ ${ }^{b}$ Dipartimento di Ingegneria Civile, Edile-Architettura e Ambientale (DICEAA), University of L'Aquila, L'Aquila, Italy \\ ${ }^{c}$ Department of Civil and Environmental Engineering (DICA), Politecnico di Milano, Milan, Italy \\ ${ }^{d}$ Institut de Mathématiques de Toulon, Université de Toulon, Toulon, France \\ ${ }^{e}$ International Research Center on Mathematics and Mechanics of Complex Systems, University of L'Aquila, L'Aquila, Italy
}

\begin{abstract}
Second-gradient continua are defined as those continua whose internal virtual work functionals depend on the first and second-gradient of the virtual displacement. These functionals can be represented either in Lagrangian (referential) or Eulerian (spatial) description thus defining respectively the Piola-Lagrange as well as the Cauchy-Euler stress and double-stress. In this paper, we deduce the Piola transformation formulae, i.e., those relationships between all Lagrangian and Eulerian fields relevant for the formulation of the Principle of Virtual Work. In particular, we derive the Piola transformations of stress and double-stress as well as the Piola transformations for external virtual work functionals compatible with second-gradient internal work functionals. The latter transformations contain in fact the Piola transformations of the contact surface and line forces as well as the contact surface double-forces.
\end{abstract}

Keywords: Continuum Mechanics, Second-Gradient Continua, Piola Transformation, Principle of Virtual Work, Lagrangian Formulation, Eulerian Formulation

\section{Introduction}

The equations of motion for three-dimensional continua are naturally deduced from the Principle of Virtual Work by using the so-called Lagrangian description. The 5 chosen space of configurations consists of the set of admissible placements, which are functions that map the position of a material particle in the reference configuration into its position in the current configuration. The reference configuration is the domain of the considered scalar, vec10 tor and tensor fields in Lagrangian description. In some applications of continuum mechanics, as for instance in fluid mechanics, it is more useful to consider fields defined on the current configuration. Also when dealing with deformable solids, externally applied forces are described 15 more naturally as vector fields defined in the current configurations of the bodies to which they are applied. It is thus of the greatest importance to to be able to use the so-called Eulerian description too. Obviously, Lagrangian and Eulerian descriptions must be fully equivalent. In20 deed, Gabrio Piola established in his fundamental works $[1,2]$ the formulas determining the relationships between ${ }_{45}$ Eulerian and Lagrangian descriptions. The obtained Piola transformations allow for the deduction of the equations

\footnotetext{
*Corresponding author

Email address: eugster@inm.uni-stuttgart.de (Simon R. $\left.\operatorname{Eugster}^{a, e, *}\right)$
}

of motion for fluids in the Eulerian description when start25

\section{Lagrangian description.}

Piola transformations of the quantities dual to the first and second gradients of the virtual displacements (i.e., stresses and double-stresses) in the internal work functionals follow from the requirement that the evaluation of the internal, external and inertial virtual work functionals should not change their value whether they are expressed in Lagrangian or in Eulerian form. In other words, the Piola transformation can be obtained by regarding the placement as a change of variables in the integral expression of the involved virtual work functionals.

Piola transformations in classical first gradient theory are well known and widely used. Similar transformations applicable for second-gradient continua are not a trivial generalization. In this paper, we determine the $\mathrm{Pi}$ ola transformations of stress and double-stress introduced in the Lagrangian configuration into their Eulerian counterparts. Moreover, we give the transformation formulae of the compatible Eulerian external virtual work functionals, which determine the relationships between the Eulerian and Lagrangian external line forces, surface forces and surface double-forces. We surprisingly find that Eulerian double-forces not only contribute to the expression of Lagrangian double-forces but also to Lagrangian line and o surface forces. 


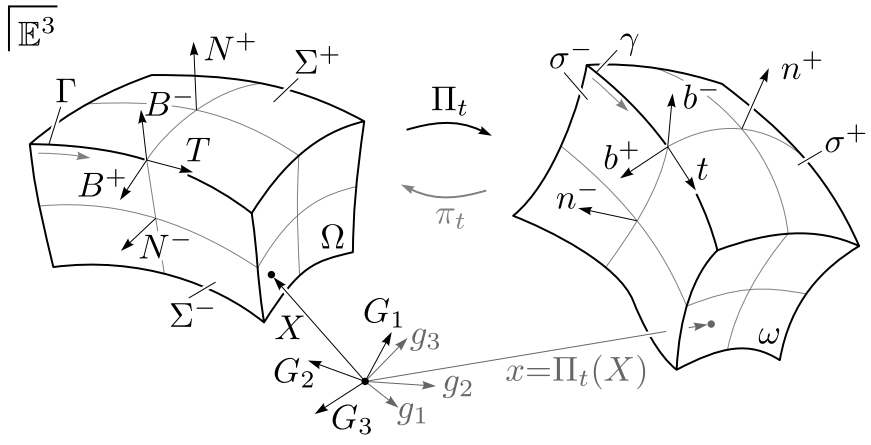

Figure 1: Reference and current configuration of a body with a piecewise continuous boundary surface and piecewise continuous boundary curves.

It has to be explicitly remarked that, to our knowledge, in the literature on second-gradient continua one can find either formulations in Eulerian description [3-7] or in Lagrangian description [8-16], but the relation between them 55 seems not to be available. For instance generalization to $N$-th gradient continua $[17,18]$ needs only the Lagrangian description. Situations are similar when homogenization methods are used to obtain the deformation energy of materials belonging to the class of higher gradient continua, 60 see among others [19-22].

\section{Lagrangian Kinematics}

The physical space is modeled as a three-dimensional Euclidean vector space $\mathbb{E}^{3}$ with the inner product denoted by $\langle\cdot, \cdot\rangle_{\mathbb{E}^{3}}$. We assume the reference configuration of the considered body $\mathcal{B}$ to be a subset $\Omega \subset \mathbb{E}^{3}$, which is sufficiently regular to perform all the required calculations, see Figure 1 for a particular example of such a configuration. The topological boundary of $\Omega$ is denoted by $\partial \Omega$. The boundary $\partial \Omega$ is the union of a finite number of twodimensional orientable surfaces with boundary, called faces of $\Omega$. The faces are oriented in accordance with their corresponding outward-pointing unit normal fields $N$. Each of the faces' boundary curves are assumed to have a piecewise continuous tangent $T$ as well as an outward-pointing unit normal $B$ that is tangent to the face. The union of all boundary curves is denoted by $\partial \partial \Omega$. Remark that each curve constituting the boundaries of the faces, which are called edges of $\Omega$, must be regarded as part of the boundary of both intersecting faces. Hence, considering Figure 1, the outward-pointing unit normal $B$ to $\partial \partial \Omega$ takes two values on the edge curve $\Gamma$. These are denoted by $B^{+}$and $B^{-}$depending on whether they are tangent to the face $\Sigma^{+}$or $\Sigma^{-}$, respectively. Edges of $\Omega$ are assumed to concur in a finite number of wedges together with a finite number of other edges. See [17] for more details about the differential-geometric assumptions accepted here.

The motion of the body $\mathcal{B}$ is defined as the mapping $\Pi: \Omega \times \mathbb{R} \rightarrow \mathbb{E}^{3},(X, t) \mapsto x=\Pi(X, t)$, which is a differentiable parametrization of suitably regular placement maps
$\Pi_{t}=\Pi(\cdot, t): \Omega \rightarrow \mathbb{E}^{3}$, which are assumed to be one to one. At some fixed time instant $t$, the image $\omega=\Pi_{t}(\Omega) \subset \mathbb{E}^{3}$ is called the current configuration of the body $\mathcal{B}$ and represents the spatial points occupied by the body $\mathcal{B}$ in its deformed state. We use the upper-case $X \in \Omega$ and the lower-case $x \in \omega$ to denote points in the reference and current configuration, respectively. Although working in $\mathbb{E}^{3}$, clever choices of representation allow for a precise classification of the appearing objects. For that reason, we use an arbitrary skew basis $\left(G_{1}, G_{2}, G_{3}\right)$ to represent the referential points as linear combination $X=X^{A} G_{A}$. Note that we apply Einstein's summation convention, which implies summation over upper and lower indices that appear twice in a term. Evaluation of the inner product for all combinations of these base vectors yields the components of the associated bilinear form, which are referred to as referential metric components $G_{A B}=\left\langle G_{A}, G_{B}\right\rangle_{\mathbb{E}^{3}}$. The points in the current configuration are represented with respect to an alternative basis $\left(g_{1}, g_{2}, g_{3}\right)$, which induces the current metric components $g_{i j}=\left\langle g_{i}, g_{j}\right\rangle_{\mathbb{E}^{3}}$. Henceforth, we refer to these two bases as referential and current basis and follow the convention to use upper- and lower-case letters also for the appearing indices. Hence, the placement map $\Pi_{t}$ is represented in such a way that

$$
x=x^{i} g_{i}=\Pi_{t}(X)=\Pi_{t}^{i}(X) g_{i} .
$$

The components of the first gradient $F=\nabla \Pi_{t}$ and the second gradient $\mathbb{F}=\nabla F=\nabla\left(\nabla \Pi_{t}\right)$ of the placement map are then given as

$$
\begin{aligned}
F_{A}^{i} & :=\left(\nabla \Pi_{t}\right)_{A}^{i}=\frac{\partial \Pi_{t}^{i}}{\partial X^{A}}, \\
\mathbb{F}_{A B}^{i} & :=(\nabla F)_{A B}^{i}=\frac{\partial^{2} \Pi_{t}^{i}}{\partial X^{A} \partial X^{B}},
\end{aligned}
$$

both of which are functions of $(X, t)$.

In order to check whether the current configuration minimizes a certain functional, the placement map $\Pi_{t}$ can be embedded in a variational family of mappings $\hat{\Pi}_{t}: \mathbb{R} \times$ $\Omega \rightarrow \mathbb{E}^{3},(\varepsilon, X) \mapsto x=\hat{\Pi}_{t}(\varepsilon, X)$, such that $\Pi_{t}(X)=$ $\hat{\Pi}_{t}(0, X)$. The variation of the placement map is then defined as

$$
\delta \Pi_{t}(X):=\frac{\partial \hat{\Pi}_{t}^{i}}{\partial \varepsilon}(0, X) g_{i}=: \delta \Pi^{i}(X, t) g_{i}
$$

inducing a virtual displacement field

$$
\delta \Pi: \Omega \times \mathbb{R} \rightarrow \mathbb{E}^{3},(X, t) \mapsto \delta \Pi^{i}(X, t) g_{i},
$$

which geometrically can be seen as a time-dependent vector field along the placement $\Pi_{t}$. Note that we use the notational convention to denote Lagrangian fields, which are defined on $\Omega$, with uppercase letters. Geometrically similar fields are the velocity and acceleration fields defined as

$$
V(X, t):=\frac{\partial \Pi^{i}}{\partial t}(X, t) g_{i}, A(X, t):=\frac{\partial^{2} \Pi^{i}}{\partial t^{2}}(X, t) g_{i} .
$$


Since the variation of the placement map induces a change in the first and the second-gradient, by the symmetry of the second derivatives, one readily sees the relations

$$
\begin{aligned}
\delta F_{A}^{i} & =\left.\frac{\partial}{\partial \varepsilon}\left(\frac{\partial \hat{\Pi}_{t}^{i}}{\partial X^{A}}\right)\right|_{\varepsilon=0}=\frac{\partial \delta \Pi^{i}}{\partial X^{A}} \\
\delta \mathbb{F}_{A B}^{i} & =\left.\frac{\partial}{\partial \varepsilon}\left(\frac{\partial^{2} \hat{\Pi}_{t}^{i}}{\partial X^{A} \partial X^{B}}\right)\right|_{\varepsilon=0}=\frac{\partial^{2} \delta \Pi^{i}}{\partial X^{A} \partial X^{B}}
\end{aligned}
$$

\section{Virtual Work Principle in Lagrangian Form}

The basic assumption in a variational formulation of continuum mechanics as founded by d'Alembert and Lagrange consists in postulating that the motion of every continuum can be characterized by suitably choosing the constitutive equations for the three linear continuous virtual work functionals $\delta \mathscr{W}_{\Omega}^{\text {int }}, \delta \mathscr{W}_{\Omega}^{\text {ext }}, \delta \mathscr{W}_{\Omega}^{\text {dyn }}$ and by assuming that, at every instant of time $t$, the virtual work identity

$$
\delta \mathscr{W}_{\Omega}^{\mathrm{tot}}(\delta \Pi):=\left(\delta \mathscr{W}_{\Omega}^{\mathrm{int}}+\delta \mathscr{W}_{\Omega}^{\mathrm{ext}}+\delta \mathscr{W}_{\Omega}^{\mathrm{dyn}}\right)(\delta \Pi)=0
$$

holds for every virtual displacement field $\delta \Pi$. The functional $\delta \mathscr{W}_{\Omega}^{\text {int }}$ captures the virtual work expended in the internal interactions among parts of the considered body $\Omega$ on every virtual deformation process. The functional $\delta \mathscr{W}_{\Omega}^{\text {ext }}$ allows for the calculation of the virtual work expended in the interactions of the body with its external world. Finally, $\delta \mathscr{W}_{\Omega}^{\text {dyn }}$ gives the inertial virtual work expended on virtual displacements.

For second-gradient continua, the internal virtual work of $\Omega$ can always be represented as the integral of a volume density in the form ${ }^{1}$

$$
\delta \mathscr{W}_{\Omega}^{\mathrm{int}}(\delta \Pi)=-\int_{\Omega}\left(P_{i}^{A} \delta F_{A}^{i}+\mathbb{P}_{i}^{A B} \delta \mathbb{F}_{A B}^{i}\right),
$$

where $P_{i}^{A}$ and $\mathbb{P}_{i}^{A B}$ are the components being work dual to the first and second gradient of the virtual displacement. We call them the Piola-Lagrange stress $P$ and Piola-Lagrange double-stress $\mathbb{P}$.

Let $\rho_{0}=\rho_{0}(X)$ be the reference density of the continuum with mass $m=\int_{\Omega} \rho_{0}$. We incorporate inertial effects by postulating the following virtual work contribution

$$
\delta \mathscr{W}_{\Omega}^{\mathrm{dyn}}(\delta \Pi)=-\int_{\Omega} \rho_{0} g_{i j} A^{j} \delta \Pi^{i},
$$

where $A^{j}$ are the components of the acceleration.

The external virtual work functional specifies the interactions between the considered continuum and its external world. Postulating the Principle of Virtual Work (7) implies that the class of possible external virtual work functionals for a continuum is limited once its internal and

\footnotetext{
${ }^{1}$ Without fixing a particular constitutive law for $P$ and $\mathbb{P}$, the sign of the virtual work expression is irrelevant.
}

inertial virtual work functionals are specified. This class is called the set of compatible external virtual work functionals. To be more specific, if one assumes an external virtual work contribution that cannot be balanced either by the internal or the inertial virtual work contributions, the Principle of Virtual Work tells us that these contributions must vanish. As external virtual work functionals include boundary interactions concentrated on $\partial \Omega$, the determination of their compatibility requires a process of integration by parts.

The internal virtual work (8) is in fact a representation of a second-order distribution in the form of (A.5). As proven in Appendix A, the internal virtual work functional can also be represented as

$$
\begin{aligned}
\delta \mathscr{W}_{\Omega}^{\mathrm{int}}(\delta \Pi)= & \int_{\Omega} \frac{\partial \bar{P}_{i}^{A}}{\partial X^{A}} \delta \Pi^{i}-\int_{\partial \Omega} \bar{P}_{i}^{A} N_{A} \delta \Pi^{i} \\
& +\int_{\partial \Omega} M_{\|}^{C} \frac{\partial}{\partial X^{C}}\left(\mathbb{P}_{i}^{A B} N_{B} M_{\|_{A}}^{L}\right) \delta \Pi^{i} \\
& -\int_{\partial \Omega}\left(\mathbb{P}_{i}^{A B} N_{A} N_{B}\right) \frac{\partial \delta \Pi^{i}}{\partial X^{C}} N^{C} \\
& -\int_{\partial \partial \Omega}\left(\mathbb{P}_{i}^{A B} B_{A} N_{B}\right) \delta \Pi^{i}
\end{aligned}
$$

where $M_{\|}$denotes the projector to the tangent spaces of $\partial \Omega$ and

$$
\bar{P}_{i}^{A}=P_{i}^{A}-\frac{\partial \mathbb{P}_{i}^{A B}}{\partial X^{B}} .
$$

Since the inertial virtual work is provided only by a volume integral, the representation (10) together with the virtual work principle (7) implies that the external virtual work functionals must be of the form

$$
\begin{aligned}
\delta \mathscr{W}_{\Omega}^{\mathrm{ext}}(\delta \Pi)= & \int_{\Omega} F_{i}^{\Omega} \delta \Pi^{i}+\int_{\partial \Omega} F_{i}^{\partial \Omega} \delta \Pi^{i} \\
& +\int_{\partial \Omega} D_{i}^{\partial \Omega} \frac{\partial \delta \Pi^{i}}{\partial X^{C}} N^{C}+\int_{\partial \partial \Omega} F_{i}^{\partial \partial \Omega} \delta \Pi^{i} .
\end{aligned}
$$

In this expression, the co-vector fields $F^{\Omega}, F^{\partial \Omega}$ and $F^{\partial \partial \Omega}$ are dual to virtual displacements and are, due to their integration domain, forces per unit reference volume, surface and line, respectively. Moreover, an additional surface density field $D^{\partial \Omega}$ appears, which, following the nomenclature used by Germain $[4,5]$, is called surface density of double-forces. These contributions are dual to the normal derivative of the virtual displacement.

Using (9) - (12) in (7), for an unconstrained continuum, the Principle of Virtual Work implies the equations of motion given by the partial differential equations

$$
\frac{\partial}{\partial X^{A}}\left(P_{i}^{A}-\frac{\partial \mathbb{P}_{i}^{A B}}{\partial X^{B}}\right)+F_{i}^{\Omega}=\rho_{0} g_{i j} A^{j} \quad \text { in } \Omega .
$$

Additionally, also the boundary conditions follow from the virtual work principle. These are on the faces

$$
\begin{aligned}
& F_{i}^{\partial \Omega}=\bar{P}_{i}^{A} N_{A}-M_{\|}^{C} \frac{\partial}{\partial X^{C}}\left(\mathbb{P}_{i}^{A B} N_{B} M_{\|_{A}^{L}}^{L}\right) \quad \text { on } \partial \Omega \\
& D_{i}^{\partial \Omega}=\mathbb{P}_{i}^{A B} N_{A} N_{B}
\end{aligned}
$$


and on the edges

$$
F_{i}^{\partial \partial \Omega}=\left(\mathbb{P}_{i}^{A B} B_{A} N_{B}\right)^{+}+\left(\mathbb{P}_{i}^{A B} B_{A} N_{B}\right)^{-} \text {on } \partial \partial \Omega .
$$

125 Recalling (A.12) from Appendix A, the symbols ()$^{ \pm}$denote the limits on the curves constituting $\partial \partial \Omega$ from the faces \pm of the quantities in the brackets.

\section{Virtual Work Principle in Eulerian Form}

From the Lagrangian point of view considered so far, all ${ }_{135}$ scalar, vector and tensor valued functions depend on referential points $X$ and time $t$. Since the placement $\Pi_{t}: \Omega \rightarrow$ $\omega$ is invertible, we can use also the Eulerian description, where the spatial points $x=\Pi_{t}(X)$ are regarded to be the independent variable. The inverse function $\pi_{t}=\Pi_{t}^{-1}: \omega \rightarrow$ $\Omega$ is written with lower case letters, as it will be done henceforth for every map with $\omega$ as its domain. Moreover, we introduce the inverse map $\pi: \omega \times \mathbb{R} \rightarrow \Omega \times \mathbb{R}$ defined as

$$
(x, t) \mapsto(X, t)=\pi(x, t):=\left(\pi_{t}(x), t\right)=\left(\Pi_{t}^{-1}(x), t\right) .
$$

This change of parameterization induces the spatial virtual displacement $\delta x$, the spatial velocity $v$ and the spatial acceleration $a$, all of which are defined via their Lagrangian counterpart as

$$
\delta x:=\delta \Pi \circ \pi, v:=V \circ \pi, a:=A \circ \pi .
$$

According to our terminology, (17) are the Piola transformations of the virtual displacement, velocity and acceleration. The components of the first and second-gradient of the spatial virtual displacement are abbreviated as

$$
\delta d_{j}^{i}:=\frac{\partial \delta x^{i}}{\partial x^{j}}, \delta \mathbb{d}_{j k}^{i}:=\frac{\partial \delta x^{i}}{\partial x^{j} \partial x^{k}} .
$$

Lagrangian and Eulerian formulations describe the same physical phenomena. For this reason, the value of the virtual work expressions should not be affected by a change of variables. Therefore

$$
\begin{aligned}
\delta \mathscr{W}_{\omega}^{\mathrm{int}}(\delta x) & :=\delta \mathscr{W}_{\Omega}^{\mathrm{int}}(\delta \Pi), \\
\delta \mathscr{W}_{\omega}^{\mathrm{ext}}(\delta x) & :=\delta \mathscr{W}_{\Omega}^{\mathrm{ext}}(\delta \Pi), \\
\delta \mathscr{W}_{\omega}^{\mathrm{dyn}}(\delta x) & :=\delta \mathscr{W}_{\Omega}^{\mathrm{dyn}}(\delta \Pi)
\end{aligned}
$$

Consequently, the virtual work equality holds also in the Eulerian description:

$$
\delta \mathscr{W}_{\omega}^{\mathrm{tot}}(\delta x):=\left(\delta \mathscr{W}_{\omega}^{\mathrm{int}}+\delta \mathscr{W}_{\omega}^{\mathrm{ext}}+\delta \mathscr{W}_{\omega}^{\mathrm{dyn}}\right)(\delta x)=0 .
$$

Since after a simple change of variables, the internal virtual work integral expression is still a representation of a second-order distribution, it can be written in the form

$$
\delta \mathscr{W}_{\omega}^{\mathrm{int}}(\delta x)=-\int_{\omega}\left(c_{i}^{j} \delta d_{j}^{i}+\mathbb{C}_{i}^{j k} \delta \mathbb{d}_{j k}^{i}\right),
$$

where $c_{i}^{j}$ and $\mathbb{C}_{i}^{j k}$ are the components of the work conjugates to the first and second gradient of the spatial virtual displacement. We call them the Cauchy-Euler stress $c$ and the Cauchy-Euler double-stress $\mathbb{C}$.

The inertial virtual work is expected to be of the form

$$
\delta \mathscr{W}_{\omega}^{\mathrm{dyn}}(\delta x)=-\int_{\omega} \rho g_{i j} a^{j} \delta x^{i},
$$

where $\rho=\rho(x, t)$ denotes the current mass density of the continuum resulting in the current inertial force density $f_{i}^{\text {dyn, } \omega}=-\rho g_{i j} a^{j}$.

To characterize the compatible external virtual work functional, the same integration by parts procedure can be applied as in the Lagrangian formulation. Defining

$$
\bar{c}_{i}^{j}=c_{i}^{j}-\frac{\partial \mathbb{C}_{i}^{j k}}{\partial x^{k}},
$$

in Appendix $\mathrm{A}$ it is proven that the Eulerian internal virtual work functional has the following representation:

$$
\begin{aligned}
& \delta \mathscr{W}_{\omega}^{\mathrm{int}}(\delta x)=\int_{\omega} \frac{\partial \bar{c}_{i}^{j}}{\partial x^{j}} \delta x^{i}-\int_{\partial \omega} \bar{c}_{i}^{j} n_{j} \delta x^{i} \\
& \quad+\int_{\partial \omega} m_{\|_{l}}^{c} \frac{\partial}{\partial x^{c}}\left(\mathbb{C}_{i}^{j k} n_{k} m_{\|_{j}}^{l}\right) \delta x^{i} \\
& \quad-\int_{\partial \omega}\left(\mathbb{C}_{i}^{j k} n_{j} n_{k}\right) \frac{\partial \delta x^{i}}{\partial x^{c}} n^{c}-\int_{\partial \partial \omega}\left(\mathbb{C}_{i}^{j k} b_{j} n_{k}\right) \delta x^{i} .
\end{aligned}
$$

Note that $n$ and $b$ denote the outward-pointing unit normals to the faces $\partial \omega$ and edges $\partial \partial \omega$ of the current configuration and $m_{\|}$the projector to the tangent spaces of $\partial \omega$. Consequently, the compatible external virtual work functionals must be of the form

$$
\begin{aligned}
\delta \mathscr{W}_{\omega}^{\mathrm{ext}}(\delta x)= & \int_{\omega} f_{i}^{\omega} \delta x^{i}+\int_{\partial \omega} f_{i}^{\partial \omega} \delta x^{i} \\
& +\int_{\partial \omega} d_{i}^{\partial \omega} \frac{\partial \delta x^{i}}{\partial x^{c}} n^{c}+\int_{\partial \partial \omega} f_{i}^{\partial \partial \omega} \delta x^{i},
\end{aligned}
$$

where the co-vector fields $f^{\omega}, f^{\partial \omega}$ and $f^{\partial \partial \omega}$ are forces per unit current volume, surface and line, respectively. Also in the Eulerian framework, there appears a surface density of double-forces $d_{i}^{\partial \omega}$, which is a density per unit current surface and which is dual to the normal gradient with respect to the current normal vector.

The Principle of Virtual Work then implies for an unconstrained continuum the governing equations of motion

$$
\frac{\partial}{\partial x^{j}}\left(c_{i}^{j}-\frac{\partial \mathbb{C}_{i}^{j k}}{\partial x^{k}}\right)+f_{i}^{\omega}=\rho g_{i j} a^{j} \quad \text { in } \omega,
$$

together with the boundary conditions on the faces

$$
\begin{aligned}
f_{i}^{\partial \omega} & =\bar{c}_{i}^{j} n_{j}-m_{\|_{l}}^{c} \frac{\partial}{\partial x^{c}}\left(\mathbb{C}_{i}^{j k} n_{k} m_{\|_{j}^{l}}^{l}\right) \text { on } \partial \omega \\
d_{i}^{\partial \omega} & =\mathbb{C}_{i}^{j k} n_{j} n_{k}
\end{aligned}
$$

and on the edges

$$
f_{i}^{\partial \partial \omega}=\left(\mathbb{C}_{i}^{j k} b_{j} n_{k}\right)^{+}+\left(\mathbb{C}_{i}^{j k} b_{j} n_{k}\right)^{-} \text {on } \partial \partial \omega .
$$




\section{Piola Transformations of Mass Density, Stress and Double-Stress}

In the previous section, we have introduced as Eulerian dual quantities the Cauchy-Euler stresses together with the current inertial force, the current external forces and current double-forces. The Piola transformation problem raises the following question: "Which are the relationships between the Lagrangian and Eulerian stresses and double-stresses, inertial forces as well as external forces and double-forces such that the identities (19) are satisfied?".

We present in this section the Piola transformation between the referential and current mass density as well as the Piola transformations between the Piola-Lagrange and the Cauchy-Euler stresses and double-stresses.

Let $\Phi$ be a time-dependent Lagrangian field with domain $\Omega$ that is related to the corresponding Eulerian field $\phi$ with domain $\omega$ by $\Phi(X, t)=\phi\left(\Pi_{t}(X), t\right)$. Recalling (2), the chain rule implies that the gradients of the Lagrangian and Eulerian fields are connected by

$$
\frac{\partial \Phi}{\partial X^{A}}(X, t)=\frac{\partial \phi}{\partial x^{j}}\left(\Pi_{t}(X), t\right) F_{A}^{j}(X, t) .
$$

Since this relation can also be written as

$$
\frac{\partial \Phi}{\partial X^{A}}(\pi(x, t))=\frac{\partial \phi}{\partial x^{j}}(x, t) F_{A}^{j}(\pi(x, t)),
$$

we will drop the arguments in what follows. The indication of the arguments may be complemented by the reader. Using this convention together with (2), we obtain, by taking once more the gradient of (29), the expression

$$
\frac{\partial^{2} \Phi}{\partial X^{A} \partial X^{B}}=\frac{\partial^{2} \phi}{\partial x^{j} \partial x^{k}} F_{A}^{j} F_{B}^{k}+\frac{\partial \phi}{\partial x^{j}} \mathbb{F}_{A B}^{j} .
$$

Consequently, the gradients of the Lagrangian and Eulerian virtual displacement fields are related by

$$
\delta F_{A}^{i}=\delta d_{j}^{i} F_{A}^{j}, \delta \mathbb{F}_{A B}^{i}=\delta \mathbb{d}_{j k}^{i} F_{A}^{j} F_{B}^{k}+\delta d_{j}^{i} \mathbb{F}_{A B}^{j} .
$$

In a similar way, the time derivative of the Lagrangian field $\Phi$ together with (5) leads to

$$
\frac{\partial \Phi}{\partial t}(X, t)=\frac{\partial \phi}{\partial x^{j}}\left(\Pi_{t}(X), t\right) V^{j}(X, t)+\frac{\partial \phi}{\partial t}\left(\Pi_{t}(X), t\right)
$$

As done in $(30)$, by using $V^{j}(\pi(x, t))=v^{j}(x, t)$, this relation can be written in spatial coordinates as

$$
\frac{\partial \Phi}{\partial t}(\pi(x, t))=\frac{\partial \phi}{\partial x^{j}}(x, t) v^{j}(x, t)+\frac{\partial \phi}{\partial t}(x, t),
$$

which then serves as definition of the material time derivative

$$
\frac{\mathrm{d}}{\mathrm{d} t} \phi(x, t):=\frac{\partial \phi}{\partial x^{j}}(x, t) v^{j}(x, t)+\frac{\partial \phi}{\partial t}(x, t) .
$$

This is why the spatial acceleration field $a$ can be expressed as the material time derivative of the spatial velocity field, which in formulas reads as

$$
a^{i}(x, t)=A^{i}(\pi(x, t))=\frac{\partial V^{i}}{\partial t}(\pi(x, t))=\frac{\mathrm{d}}{\mathrm{d} t} v^{i}(x, t) .
$$

To obtain the transformation formulae between referential and spatial fields, we will take the Lagrangian virtual work expressions (8) and (9) and change the integration variable from the referential coordinates $X$ to the spatial coordinates $x$ resulting in an integral expression defined on the domain $\omega$.

Transforming the right hand side of (8), by the change of variables given by $\pi_{t}(x)$, whose Jacobian determinant is equal to $J^{-1}(\pi(x, t))=\operatorname{det}(F(\pi(x, t)))^{-1}$, we get with the above introduced abuse of notation

$$
\delta \mathscr{W}_{\Omega}^{\mathrm{int}}(\delta \Pi)=-\int_{\omega} J^{-1}\left(P_{i}^{A} \delta F_{A}^{i}+\mathbb{P}_{i}^{A B} \delta \mathbb{F}_{A B}^{i}\right)
$$

The integration domain $\omega$ makes it clear that all capitalized Lagrangian fields must be composed with $\pi$. Next, we insert the relations (32) and compare the outcome with the Eulerian virtual work expression (21) in the following way

$$
\begin{aligned}
\delta \mathscr{W}_{\Omega}^{\text {int }}(\delta \Pi)= & -\int_{\omega} J^{-1} P_{i}^{A} \delta d_{j}^{i} F_{A}^{j} \\
& -\int_{\omega} J^{-1} \mathbb{P}_{i}^{A B}\left(\delta \mathbb{d}_{j k}^{i} F_{A}^{j} F_{B}^{k}+\delta d_{j}^{i} \mathbb{F}_{A B}^{j}\right) \\
= & -\int_{\omega} J^{-1}\left(P_{i}^{A} F_{A}^{j}+\mathbb{P}_{i}^{A B} \mathbb{F}_{A B}^{j}\right) \delta d_{j}^{i} \\
& -\int_{\omega} J^{-1}\left(\mathbb{P}_{i}^{A B} F_{A}^{j} F_{B}^{k}\right) \delta \mathbb{d}_{j k}^{i} \\
= & -\int_{\omega}\left(c_{i}^{j} \delta d_{j}^{i}+\mathbb{C}_{i}^{j k} \delta \mathbb{d}_{j k}^{i}\right)=\delta \mathscr{W}_{\omega}^{\operatorname{int}}(\delta x) .
\end{aligned}
$$

Comparison of the last and second last line leads to the Piola transformation of stress and double-stress

$$
\begin{aligned}
c_{i}^{j} & =J^{-1}\left(P_{i}^{A} F_{A}^{j}+\mathbb{P}_{i}^{A B} \mathbb{F}_{A B}^{j}\right), \\
\mathbb{C}_{i}^{j k} & =J^{-1} \mathbb{P}_{i}^{A B} F_{A}^{j} F_{B}^{k},
\end{aligned}
$$

which can be written in a more synthetic way $\mathrm{as}^{2}$

$$
\begin{aligned}
c & =J^{-1}\left(P \cdot F^{t}+\mathbb{P}: \mathbb{F}^{t}\right), \\
\mathbb{C} & =J^{-1} \mathbb{P}:\left(F^{t} \bar{\otimes} F^{t}\right) .
\end{aligned}
$$

Note that with our notation, either the left hand sides have to be composed with $\Pi$ or the right hand sides with $\pi$. The reader will remark that, by simply assuming $\mathbb{P}=0$, we get the Piola transformation formula $c=\left(J^{-1} P \cdot F^{t}\right) \circ \pi$ for first gradient continua.

Transforming in the same way as before the Lagrangian inertial virtual work expression, we get

$$
\delta \mathscr{W}_{\Omega}^{\mathrm{dyn}}(\delta \Pi)=-\int_{\omega} \rho_{0} g_{i j} A^{j} \delta \Pi^{i} J^{-1}
$$

${ }^{2}$ Note the non-standard operators required for the higher order tensors. Using the vectors $a, b \in \mathbb{E}^{3}$, the co-vectors $\alpha, \beta \in\left(\mathbb{E}^{*}\right)^{3}$ and the third order tensor $\mathbb{A} \in \otimes_{2}^{1} \mathbb{E}^{3}$, these operators can be defined by the equalities $(\alpha \otimes a) \bar{\otimes}(\beta \otimes b)=(\alpha \otimes \beta) \otimes(a \otimes b)$ and $\alpha \cdot \mathbb{A}:(a \otimes b)=$ $(a \otimes b): \mathbb{A}^{t} \cdot \alpha$. The remaining operators are standard and can be recovered by comparing (40) with (39). 
Using the definition of the spatial virtual displacement and acceleration from (17), we can further manipulate the expression to

$$
\begin{aligned}
\delta \mathscr{W}_{\Omega}^{\mathrm{dyn}}(\delta \Pi) & =-\int_{\omega} \rho_{0} J^{-1} g_{i j} a^{j} \delta x^{i} \\
& =-\int_{\omega} \rho g_{i j} a^{j} \delta x^{i}=\delta \mathscr{W}_{\omega}^{\mathrm{dyn}}(\delta x),
\end{aligned}
$$

where we recognize in the last step the relation between referential and current mass density. This Piola transformation of the density expressed in Lagrangian coordinates is generally referred to as referential equation of continuity and reads as

$$
\rho_{0}(X)=J(X, t) \rho(\Pi(X, t), t) .
$$

The spatial equation of continuity

$$
\frac{\mathrm{d}}{\mathrm{d} t} \rho(x, t)+\rho(x, t) \frac{\partial v^{i}}{\partial x^{i}}=0
$$

follows immediately by carrying out the following computations. First, take the time derivative of (43), then insert the identity $\partial J / \partial t=J \partial v^{i} / \partial x^{i}$ and, last, express the result in spatial coordinates with the spatial velocity field and utilize the material time derivative (35).

\section{Piola Transformations of external forces and double-forces}

The final relations left to show are the Piola transformations of the forces and double-forces appearing in the Lagrangian and Eulerian external virtual work functionals (12) and (25). We proceed in a similar way as just done for the stresses and double-stresses. However, some further unexpected difficulties arise. Carrying out the change of variable given by the inverse of the placement, the Eulerian normal derivative to $\partial \omega$ of the virtual displacement does not transform into the Lagrangian normal derivative to $\partial \Omega$. As a consequence, the Eulerian virtual work functional of double-forces, once transformed into Lagrangian coordinates followed by a Lagrangian surface integration by parts, is composed of three virtual work functionals: a first one to be identified with the Lagrangian virtual work functional of double-forces, a second one contributing to Lagrangian surface forces and the last one contributing to Lagrangian edge forces.

Using the inverse of the right Cauchy-Green strain $C^{-1}$ with the components

$$
\left(C^{-1}\right)^{A B}=\left(F^{-1}\right)_{i}^{A} g^{i j}\left(F^{-1}\right)_{j}^{B},
$$

as well as

$$
\begin{aligned}
K^{A} & =M_{\|_{C}^{A}}^{A}\left(C^{-1}\right)^{C B} N_{B} \\
& =\left(\left(F^{-1}\right)_{i}^{A} g^{i j}\left(F^{-1}\right)_{j}^{B}-\left\|F^{-T} \cdot N\right\|^{2} G^{A B}\right) N_{B},
\end{aligned}
$$

we get after long but straightforward calculations the following transformation formulae for the external forces:

$$
\begin{aligned}
F_{i}^{\Omega}= & J f_{i}^{\omega}, \\
F_{i}^{\partial \Omega}= & \left\|J F^{-T} \cdot N\right\| f_{i}^{\partial \omega}-M_{\|_{A}^{B}}^{B} \frac{\partial}{\partial X^{B}}\left(J d_{i}^{\partial \omega} K^{A}\right), \\
F_{i}^{\partial \partial \Omega}= & \|F \cdot T\| f_{i}^{\partial \partial \omega}+\left(J\left(C^{-1}\right)^{A B} B_{A} N_{B} d_{i}^{\partial \omega}\right)^{+} \\
& +\left(J\left(C^{-1}\right)^{A B} B_{A} N_{B} d_{i}^{\partial \omega}\right)^{-} .
\end{aligned}
$$

Using definition (A.3), we can write the Piola transformations of the external forces in direct notation as

$$
\begin{aligned}
F^{\Omega}= & J f^{\omega}, \\
F^{\partial \Omega}= & \left\|J F^{-T} \cdot N\right\| f^{\partial \omega}-\operatorname{div}_{\|}^{\partial \Omega}\left(J d^{\partial \omega} \otimes K\right), \\
F^{\partial \partial \Omega}= & \|F \cdot T\| f^{\partial \partial \omega}+\left(J\left(B \cdot C^{-1} \cdot N\right) d^{\partial \omega}\right)^{+} \\
& +\left(J\left(B \cdot C^{-1} \cdot N\right) d^{\partial \omega}\right)^{-} .
\end{aligned}
$$

Finally, the Piola transformation of the surface doubleforce, once in index notation and once in direct notation, reads as

$$
D_{i}^{\partial \Omega}=J\left\|F^{-T} \cdot N\right\|^{2} d_{i}^{\partial \omega}, D^{\partial \Omega}=J\left\|F^{-T} \cdot N\right\|^{2} d^{\partial \omega} .
$$

\section{Appendix A. Equivalent form for second-order dis- tributions}

Let us consider a regular manifold $\mathcal{V}$ embedded in an $n$ dimensional Euclidean vector space $\mathbb{E}^{n}$ and the idempotent projectors $m_{\|}$and $m_{\perp}$ on its tangent and normal spaces. When $\mathcal{V}$ has co-dimension one, and if $n$ denotes its unit normal vector, we have

$$
m_{\perp}^{\gamma}=n^{\gamma} n_{\alpha}, m_{\|_{\alpha}^{\gamma}}^{\gamma}=\delta_{\alpha}^{\gamma}-n^{\gamma} n_{\alpha}
$$

Given a vector field $w$ defined in the neighborhood of $\mathcal{V}$, the divergence theorem for Riemannian submanifolds with boundaries is stated as

$$
\begin{aligned}
& \int_{\mathcal{V}} m_{\|_{\alpha}^{\gamma}}^{\gamma} \frac{\partial}{\partial x^{\gamma}}\left(m_{\|_{\beta}^{\alpha}}^{\alpha} w^{\beta}\right) \\
& =\int_{\partial \mathcal{V}}\left(m_{\|_{\beta}^{\alpha}}^{\alpha} w^{\beta}\right) m_{\|_{\alpha}^{\gamma}}^{\gamma} b_{\gamma}=\int_{\partial \mathcal{V}} w^{\beta} b_{\beta},
\end{aligned}
$$

where $\partial \mathcal{V}$ denotes the boundary of $\mathcal{V}$ and where the unit vector $b$ is tangent to $\mathcal{V}$ and normal to $\partial \mathcal{V}$. Defining $\operatorname{div} \mathcal{V}$ by setting for all smooth fields $\phi$

$$
\left(\operatorname{div}_{\|}^{\mathcal{V}}(\phi)\right)_{\alpha}:=m_{\|_{\alpha}}^{\gamma} \frac{\partial}{\partial x^{\gamma}}(\phi),
$$

the divergence theorem reads as

$$
\int_{\mathcal{V}} \operatorname{div}_{\|}^{\mathcal{V}}\left(m_{\|} \cdot w\right)=\int_{\partial \mathcal{V}} w \cdot b .
$$


In accordance with the theory of distribution, both the virtual work expression in Lagrangian and Eulerian description can be considered as distributions $\mathscr{D}$ represented in the form

$$
\mathscr{D}(\phi)=\int_{v}\left(s^{\alpha} \frac{\partial \phi}{\partial y^{\alpha}}+\mathbb{s}^{\alpha \beta} \frac{\partial^{2} \phi}{\partial y^{\alpha} \partial y^{\beta}}\right),
$$

where the derivatives of the test functions $\phi$ are taken with respect to the coordinates $y^{\alpha}$ of a 3-dimensional Euclidean space. Note that the index $i$ appearing in both (8) and (21) does not play any role in the present considerations and is therefore omitted. Since the order of a distribution can be defined as the sum of the highest order derivative of the test function and the co-dimension of the integration domain in the domain of the test functions, $\mathscr{D}$ is a second-order distribution. The symbol $v$ denotes the generic integration domain which satisfies the same continuity requirements as discussed in Section 2 for the reference configuration $\Omega$. The faces of the subset $v$ are denoted by $\partial v$ and come along with the outward-pointing unit normal field $n$. The symbol $\partial \partial v$ denotes the edges on which the outward-pointing unit normals $b$ to the boundaries $\partial \partial v$ are defined. Moreover, these normals are in the tangent planes to the faces constituting $\partial v$.

Using the product rule in the second integrand of (A.5), we can write

$$
\mathscr{D}(\phi)=\int_{v}\left(s^{\alpha}-\frac{\partial \mathbb{s}^{\alpha \beta}}{\partial y^{\beta}}\right) \frac{\partial \phi}{\partial y^{\alpha}}+\int_{v} \frac{\partial}{\partial y^{\beta}}\left(\mathbb{s}^{\alpha \beta} \frac{\partial \phi}{\partial y^{\alpha}}\right) .
$$

With the abbreviation $\bar{s}^{\alpha}=s^{\alpha}-\partial \Phi^{\alpha \beta} / \partial y^{\beta}$ and applying the product rule for the first integrand, we end up with

$$
\mathscr{D}(\phi)=\int_{v} \frac{\partial}{\partial y^{\alpha}}\left(\bar{s}^{\alpha} \phi\right)-\int_{v} \frac{\partial \bar{s}^{\alpha}}{\partial y^{\alpha}} \phi+\int_{v} \frac{\partial}{\partial y^{\beta}}\left(\Phi^{\alpha \beta} \frac{\partial \phi}{\partial y^{\alpha}}\right) .
$$

Using the divergence theorem for the first and the third term and introducing the zeroth- and first-order distributions

$$
\mathscr{D}_{v}^{0}(\phi)=-\int_{v} \frac{\partial \bar{s}^{\alpha}}{\partial y^{\alpha}} \phi, \mathscr{D}_{\partial v}^{0}(\phi)=\int_{\partial v} \bar{s}^{\alpha} n_{\alpha} \phi,
$$

the distribution (A.5) can be written in the form

$$
\mathscr{D}(\phi)=\mathscr{D}_{v}^{0}(\phi)+\mathscr{D}_{\partial v}^{0}(\phi)+\int_{\partial v} \mathbb{S}^{\alpha \beta} \frac{\partial \phi}{\partial y^{\alpha}} n_{\beta} .
$$

The last term here, is the only expression in which still derivatives of $\phi$ appear. Therefore, we will manipulate this term further by using the projectors (A.1) for the faces $\partial v$

$$
\begin{aligned}
\int_{\partial v} \mathbb{S}^{\alpha \beta} \frac{\partial \phi}{\partial y^{\alpha}} n_{\beta}=\int_{\partial v} \mathbb{S}^{\alpha \beta} \frac{\partial \phi}{\partial y^{\gamma}} n_{\beta} \delta_{\alpha}^{\gamma} \\
=\int_{\partial v} \mathbb{S}^{\alpha \beta} \frac{\partial \phi}{\partial y^{\gamma}} n_{\beta}\left(m_{\|_{\alpha}^{\gamma}}^{\gamma}+m_{\perp}^{\gamma}\right) \\
=\int_{\partial v} \mathbb{S}^{\alpha \beta} \frac{\partial \phi}{\partial y^{\gamma}} n_{\beta} m_{\|_{\alpha}^{\gamma}}^{\gamma}+\mathscr{D}_{\partial v}^{I}(\phi),
\end{aligned}
$$

where we have introduced the distribution

$$
\mathscr{D}_{\partial v}^{I}(\phi)=\int_{\partial v}\left(\Phi^{\alpha \beta} n_{\alpha} n_{\beta}\right) \frac{\partial \phi}{\partial y^{\gamma}} n^{\gamma} .
$$

The distribution $\mathscr{D}_{\partial v}^{I}$ is a second-order transverse distribution involving the normal derivative of the test function $\left(\partial \phi / \partial y^{\gamma}\right) n^{\gamma}$ and cannot be reduced any further.

Due to the idempotence of the projector $m_{\|}$and by applying once more the product rule, we can manipulate the first term in the last line of (A.8) in the following way

$$
\begin{gathered}
\int_{\partial v}\left(\mathbb{S}^{\alpha \beta} \frac{\partial \phi}{\partial y^{\gamma}} n_{\beta} m_{\|_{\alpha}^{\lambda}}^{\lambda}\right) m_{\|_{\lambda}^{\gamma}}^{\gamma} \\
=\int_{\partial v}\left\{\frac{\partial}{\partial y^{\gamma}}\left(\mathbb{S}^{\alpha \beta} n_{\beta} m_{\|_{\alpha}^{\lambda}}^{\lambda} \phi\right) m_{\|_{\lambda}}^{\gamma}\right. \\
\left.-m_{\|_{\lambda}^{\gamma}}^{\gamma} \frac{\partial}{\partial y^{\gamma}}\left(\mathbb{S}^{\alpha \beta} n_{\beta} m_{\|_{\alpha}^{\lambda}}^{\lambda}\right) \phi\right\} \\
=\mathscr{D}_{\partial \partial v}^{0}(\phi)+\tilde{\mathscr{D}}_{\partial v}^{0}(\phi) .
\end{gathered}
$$

In the last step, we have introduced the second- and firstorder distributions

$$
\begin{aligned}
\mathscr{D}_{\partial \partial v}^{0}(\phi) & =\int_{\partial \partial v}\left(\mathbb{S}^{\alpha \beta} n_{\beta} b_{\alpha}\right) \phi, \\
\tilde{\mathscr{D}}_{\partial v}^{0}(\phi) & =-\int_{\partial v} m_{\|_{\lambda}^{\gamma}}^{\gamma} \frac{\partial}{\partial y^{\gamma}}\left(\mathbb{S}^{\alpha \beta} n_{\beta} m_{\|_{\alpha}^{\lambda}}^{\lambda}\right) \phi .
\end{aligned}
$$

Note, to obtain $\mathscr{D}_{\partial \partial v}^{0}$, the divergence theorem (A.2) has been applied leading to a line integral along the edges of $v$. We used here a notational convention in the expression of the integral. As depicted in Figure 1, we observe that an edge $\gamma$ is the intersection of two subsurfaces $\sigma^{+}$ and $\sigma^{-}$, say. Hence, $\gamma$ is traversed twice: once with the surface normal $n^{-}$, edge normal $b^{-}$and the limit $\left(\mathbb{S}^{-}\right)^{\alpha \beta}$ approached from the surface $\sigma^{-}$, as well as once with the corresponding $n^{+}, b^{+}$and $\left(\mathbb{s}^{+}\right)^{\alpha \beta}$. Consequently, if we denote each of the edge curves by $\gamma_{i}$ for $i=1, \ldots, n_{\mathrm{e}}$, then the integral expression of the first equality in (A.11) reads $\mathrm{as}^{3}$

$$
\sum_{i=1}^{n_{\mathrm{e}}} \int_{\gamma_{i}}\left[\left(\mathbb{S}^{\alpha \beta} n_{\beta} b_{\alpha}\right)^{+}+\left(\mathbb{S}^{\alpha \beta} n_{\beta} b_{\alpha}\right)^{-}\right] \phi
$$

In conclusion, from the point of view of the theory of distributions, the second-order distribution $\mathscr{D}(\phi)$ from (A.5) can equivalently be represented as

$$
\mathscr{D}=\mathscr{D}_{v}^{0}+\left(\mathscr{D}_{\partial v}^{0}+\tilde{\mathscr{D}}_{\partial v}^{0}\right)+\mathscr{D}_{\partial v}^{I}+\mathscr{D}_{\partial \partial v}^{0} .
$$

This equivalence can be applied to the Lagrangian infollowing replacements in (A.6), (A.9) and (A.11): $s^{\alpha} \mapsto$

\footnotetext{
${ }^{3}$ It is also common to introduce $\bar{b}^{ \pm}=t \times n^{ \pm}$as the vector product between tangent vector to the curve $\gamma$ and the surface normal $n$, see $[3,5,24]$. In that case $b^{-}=-\bar{b}^{-}$and the sign changes in the second term of (A.12)
} 
$-P_{i}^{A}, \mathbb{S}^{\alpha \beta} \mapsto-\mathbb{P}_{i}^{A B}, \bar{s}^{\alpha} \mapsto-\bar{P}_{i}^{A}, \phi \mapsto \delta \Pi^{i}, y^{\alpha} \mapsto X_{, 280}^{A}$ $v \mapsto \Omega, b \mapsto B$ and $n \mapsto N$ with the corresponding projector $m_{\|} \mapsto M_{\|}$. Remark the now appearing implicit summation over the index $i$, which has no influence on the derivations presented above. As a consequence of (A.13),285 the Lagrangian internal virtual work functional can then also be represented by (10). The following replacements are required in (A.6), (A.9) and (A.11) to obtain for the Eulerian internal virtual work functional the representa-290 tion (24): $s^{\alpha} \mapsto-c_{i}^{j}, \mathbb{S}^{\alpha \beta} \mapsto-\mathbb{C}_{i}^{j k}, \bar{s}^{\alpha} \mapsto-\bar{c}_{i}^{j}, \phi \mapsto \delta x^{i}$, $y^{\alpha} \mapsto x^{j}$ as well as $v \mapsto \omega$.

Acknowledgment. The paper was published with the financial support of the Ministry of Higher Education and ${ }^{295}$ Science of the Russian Federation as part of the program of the Moscow Center for Fundamental and Applied Mathematics under the agreement No. 075-15-2019-1621.

\section{References}

[1] F. dell'Isola, G. Maier, U. Perego, U. Andreaus, R. Esposito, S. Forest (Eds.), The complete works of Gabrio Piola: Volume I: Commented English translation, Advanced Structured Materials, Springer, 2014.

[2] F. dell'Isola, U. Andreaus, A. Cazzani, R. Esposito, L. Placidi, U. Perego, G. Maier, P. Seppecher (Eds.), The complete works ${ }_{310}$ of Gabrio Piola: Volume II: Commented English translation, Springer, 2019.

[3] R. A. Toupin, Elastic materials with couple-stresses, Archive of Rational Mechanics and Analysis 11 (1962) 385-414.

[4] P. Germain, La méthodes des puissances virtuelles en mécanique ${ }_{315}$ des milieux continus - 1ère Partie, Théorie du second gradient, Journal de Mécanique 12 (1973) 235-274.

[5] P. Germain, The method of virtual power in the mechanics of continuous media, I: Second-gradient theory, Mathematics and Mechanics of Complex Systems 8 (2) (2020) 153-190, translated ${ }_{320}$ by M. Epstein and R. E. Smelser.

[6] P. Casal, La théorie du second gradient et la capillarité, Comptes Rendus de l'Académie des Sciences Paris 274 (22) (1972) 1571-1574.

[7] P. Casal, H. Gouin, Relation entre l'équation de l'énergie et l'équation du mouvement en théorie de Korteweg de la capillarité, Comptes Rendus de l'Académie des Sciences Paris 300 (7) (1985) 231-234.

[8] N. Auffray, F. dell'Isola, V. A. Eremeyev, A. Madeo, G. Rosi, Analytical continuum mechanics à la Hamilton-Piola least action principle for second gradient continua and capillary fluids, Mathematics and Mechanics of Solids 20 (4) (2015) 375-417.

[9] P. Seppecher, Microscopic interpretation of strain-gradient and generalized continuum models, in: A. Bertram, S. Forest (Eds.), Mechanics of Strain Gradient Materials, Springer, 2020, pp. 7199.

[10] I. Giorgio, A. Ciallella, D. Scerrato, A study about the impact of the topological arrangement of fibers on fiber-reinforced composites: Some guidelines aiming at the development of new ultra-stiff and ultra-soft metamaterials, International Journal of Solids and Structures 203 (2020) 73-83.

[11] I. Giorgio, Lattice shells composed of two families of curved Kirchhoff rods: an archetypal example, topology optimization of a cycloidal metamaterial, Continuum Mechanics and Thermodynamics (2020) 1-20.

[12] I. Giorgio, M. Spagnuolo, U. Andreaus, D. Scerrato, A. M. Bersani, In-depth gaze at the astonishing mechanical behavior of bone: A review for designing bio-inspired hierarchical metamaterials, Mathematics and Mechanics of Solids 26 (7) (2020) 1074-1103.
[13] D. Scerrato, A. M. Bersani, I. Giorgio, Bio-inspired design of a porous resorbable scaffold for bone reconstruction: A preliminary study, Biomimetics 6 (18) (2021) 1-20.

[14] L. Placidi, E. Barchiesi, E. Turco, N. L. Rizzi, A review on 2D models for the description of pantographic fabrics, Zeitschrift für angewandte Mathematik und Physik 67 (5) (2016) 121:120.

[15] L. Placidi, E. Barchiesi, A. Misra, A strain gradient variational approach to damage: a comparison with damage gradient models and numerical results, Mathematics and Mechanics of Complex Systems 6 (2) (2018) 77-100.

[16] E. Turco, E. Barchiesi, Equilibrium paths of Hencky pantographic beams in a three-point bending problem, Mathematics and Mechanics of Complex Systems 7 (4) (2019) 287-310.

[17] F. dell'Isola, P. Seppecher, A. Madeo, How contact interactions may depend on the shape of Cauchy cuts in Nth gradient continua: approach "à la D'Alembert", Zeitschrift für angewandte Mathematik und Physik 63 (6) (2012) 1119-1141.

[18] F. dell'Isola, P. Seppecher, L. Placidi, E. Barchiesi, A. Misra, Least action and virtual work principles for the formulation of generalized continuum models, in: F. dell'Isola, D. J. Steigmann (Eds.), Discrete and Continuum Models for Complex Metamaterials, Cambridge University Press, 2020, pp. 327-394.

[19] E. Barchiesi, S. R. Eugster, F. dell'Isola, F. Hild, Large inplane elastic deformations of bi-pantographic fabrics: asymptotic homogenization and experimental validation, Mathematics and Mechanics of Solids 25 (3) (2020) 739-767.

[20] E. Barchiesi, F. dell'Isola, F. Hild, P. Seppecher, Twodimensional continua capable of large elastic extension in two independent directions: Asymptotic homogenization, numerical simulations and experimental evidence, Mechanics Research Communications 103 (2020) 103466:1-5.

[21] F. dell'Isola, I. Giorgio, M. Pawlikowski, N. L. Rizzi, Large deformations of planar extensible beams and pantographic lattices: heuristic homogenization, experimental and numerical examples of equilibrium, Proceedings of the Royal Society of London A: Mathematical, Physical and Engineering Sciences 472 (2016) 20150790:1-23.

[22] J.-J. Alibert, P. Seppecher, F. dell'Isola, Truss modular beams with deformation energy depending on higher displacment gradient, Mathematics and Mechanics of Solids 8 (1) (2003) 51-73.

[23] S. R. Eugster, F. dell'Isola, D. Steigmann, Continuum theory for mechanical metamaterials with a cubic lattice substructure, Mathematics and Mechanics of Complex Systems 7 (1) (2019) $75-98$. 